\title{
Comparaison randomisee en double insu d'in- jections intracaverneuses d'alprostadil-alfadex et de chlorhydrate de moxisylyte dans 156 cas de dysfonction erectile chronique (*)
}

\author{
J. Buvat 1 , E. Amar², O.Cambau ${ }^{3}$, P.Desvaux ${ }^{4}$, A. Gout5, A. Leriche 3 \\ 1 Association EPARP, 49 rue de la Bassée, Lille, France \\ 2 C.H.U. Bichat Claude-Bernard, Service d'Urologie, 46 rue Henri Huchard, Paris, France \\ 3 Service d'Urologie, Hôpital Henri Gabriel, Saint Genis Laval, France \\ 4 Hôpital Cochin, Clichy, France \\ 5 Polyclinique St Claude, Bd Schweitzer, Saint Quentin, France
}

\section{RESUME}

Buts : Comparer l'efficacité et la tolérance de l'Alprostadil-Alfadex et du chlorhydrate de Moxisylyte pour l'induction d'érections compatibles avec un rapport sexuel dans le cadre d'une étude prospective, randomisée, en double insu, avec groupes parallèles, chez des sujets avec dysfonction érectile.

Méthode : 156 sujets avec dysfonction érectile d'origine organique, non-organique, ou mixte, ont été répartis de façon randomisée en 2 groupes parallèles chez lesquels la dose optimale individuelle d'Alprostadil-Alfadex ou de chlorhydrate de Moxisylyte a été recherchée au cabinet. La réponse érectile a été mesurée à l'aide du «Buckling Test». Nous avons considéré que le test était positif en cas d'érection dont la rigidité axiale ne se déformait pas (ne pliait pas) sous l'effet d'une charge de un kilogramme. Le «buckling test» fut répété toutes les 10 minutes pendant jusque 60 minutes lorsqu'il était positif. Les sujets purent ensuite faire 6 auto-injections à domicile.

Résultats : 56 sujets du groupe AlprostadilAlfadex (75 \%) et 32 sujets du groupe chlorhydrate de Moxisylyte (40 \%) eurent au moins un buckling test positif au cabinet. Les investigateurs jugèrent les érections induites compatibles avec un rapport sexuel chez 61 sujets du groupe Alprostadil-Alfadex (81 \%) contre 37 sujets du groupe chlorhydrate de Moxisylyte (46\%). Tous les paramètres évaluant l'efficacité au cabinet atteignirent une signification statistique avec un $p<0.001$ en faveur de l'Alprostadil-Alfadex. Pendant la phase d'auto-injection à domicile, 58 sujets du groupe Alprostadil-Alfadex (85 \%) rapportèrent avoir eu au moins une érection rigide, par comparaison à 37 sujets du groupe chlorhydrate de Moxisylyte (61\%). Tant l'opinion des patients que celle de leurs partenaires sur l'intérêt du traitement atteignirent des scores significativement supérieurs dans le groupe Alprostadil-Alfadex, par comparaison au groupe chlorhydrate de Moxisylyte.

\footnotetext{
* Liste des investigateurs ayant participé à l'étude : E. Amar, L. Boccon-Gibbod, Paris ; D. Morlier, G. Arvis, Paris ; J. Buvat, A. Lemaire, Lille ; O. Cambau, A. Leriche, Saint Genis Laval ; D. Chevallier, Nice ; P. Costa, H. Navratil, Nimes ; P. Desvaux, Clichy ; J. Hermabessière, Clermont-Ferrand ; F. Giuliano, A. Jardin, Le Kremlin Bicêtre, P. Lansiaux, Paris ; B. Dufour, Paris ; M. Schouman, Neuilly ; A. Gout, H. Zerbib, R. Debré, Paris

* Ce texte est la version française de : Double-blind multicenter study comparing Alprostadil Alfadex with moxysylyte chlorhydrate in patients with chronic erectile dysfunction . J.BUVAT et al., Journal of Urology, 1998, V.159, 116-119.
} 
Conclusions : L'Alprostadil-Alfadex est significativement plus efficace que le chlorhydrate de Moxisylyte pour induire une rigidité complète tant au cabinet qu'à domicile. Les effets indésirables liés au processus d'injection surviennent avec la même fréquence avec les 2 substances, tandis que le Moxisylyte induit plus d'effets indésirables systémiques, et la PGE1 plus de douleurs et d'érections prolongées. Tant les patients que leurs partenaires sont significativement plus satisfaits de l'utilisation de l'Alprostadil-Alfadex.

Mots clés : Impuissance, dysfonction érectile, rigidité pénienne, buckling test, traitement pharmacologique, auto-injections, Prostaglandine E1, Alprostadil-Alfadex, Moxisylyte

Depuis l'introduction du traitement par injections intracaverneuses au début des années 1980 , différentes substances vaso-actives ont été testées et utilisées comme traitement des dysfonctions érectiles [2, 9, 17]. Le Moxisylyte (ICAVEX $\left.{ }^{\circledR}, E R E C N O S ®\right)$ fut la première substance au monde à recevoir l'autorisation de mise sur le marché dans cette indication [6]. L'Alprostadil fut introduit comme traitement des dysfonctions érectiles en 1986 [8, 16]. Il est actuellement considéré dans la plupart des pays comme le produit de référence pour le traitement par injections intracaverneuses, ayant également reçu l'autorisation de mise sur le marché dans plusieurs pays (CEDEX®), et CAVERJECT®, en France). Il a été montré que tant le Moxisylyte que l'Alprostadil diminuaient l'incidence des complications typiquement associées à l'usage de la papavérine et de l'association papavérine-phentolamine, soit les érections prolongées et la fibrose des corps caverneux $[3,4,5,13,15]$. L'Alprostadil-Alfadex (PGE1 $\alpha$-cd) est une formulation originale de Prostaglandine E1 (PGE1) constituée par un complexe de PGE1 et d'alphacyclodextrine (Alfadex), un oligomère de glucose cyclique. Nous rapportons ici les résultats de la première étude en double insu ayant comparé ces deux substances qui ont obtenu l'autorisation de mise sur le marché pour traiter les dysfonctions érectiles.

\section{MATERIELS ET METHODES}

\section{Schéma de l'étude}

Cette étude multicentrique impliqua 13 centres français. Son protocole fut approuvé par le Comité Consultatif de Protection des Personnes dans la Recherche Biomédicale de Valenciennes. Elle inclut des hommes atteints de dysfonction érectile chronique, après qu'ils aient signé un consentement éclairé. L'étiologie de la dysfonction érectile fut classée par chaque investigateur comme principalement organique, principalement psychogène, ou mixte en fonction de différentes explorations ayant comporté selon les cas la pléthysmographie nocturne avec monitorage de la rigidité par le Rigiscan, les tests d'injection intracaverneuse, le pharmacodoppler ou l'échodoppler pulsé après injection intracaverneuse, la cavernométrie dynamique après injection intracaverneuse, la mesure du temps de latence du réflexe bulbo-caverneux et les potentiels évoqués somesthésiques des nerfs honteux internes. Ont été pratiqués de façon systématique, en accord avec le protocole de l'étude, une évaluation biologique comportant des dosages de testostérone et de prolactine sériques, l'étude des antécédents et de l'histoire de l'affection, incluant des questions spécifiques sur la dysfonction érectile, et un examen clinique comportant tout particulièrement l'examen du pénis.

Les principaux critères d'inclusion furent un âge de 18 à 70 ans, une dysfonction érectile datant d'au moins 6 mois, et la signature d'un consentement éclairé. Les principaux critères d'exclusion furent l'éthylisme ou la toxicomanie, l'existence d'autres maladies graves, particulièrement angor instable ou infarctus $d u$ myocarde au cours des 3 mois précédant le début de l'étude, et les dysfonctions érectiles résultant de pathologies urologiques telles que l'hypospadias, les dysfonctions érectiles d'origine hormonale, et les traitements concomittants par d'autres agents vaso-actifs.

Tous les patients remplissant les critères d'inclusion et d'exclusion furent répartis de façon randomisée en 2 groupes parallèles chez lesquels la dose minima efficace fut recherchée au cabinet, dans l'un avec du Moxisylyte, en 
commençant par $5 \mathrm{mg}$ et en augmentant si nécessaire à 10 puis $20 \mathrm{mg}$, et dans l'autre avec de l'Alprostadil-Alfadex, en commençant par $5 \mu \mathrm{g}$ et en augmentant si nécessaire à 10 et au maximum $20 \mu \mathrm{g}$. Au cours de cette phase, le double insu fut permis par l'intervention d'une tierce personne indépendante (pharmacien ou médecin indépendant de l'étude), qui prépara les injections dans des seringues identiques. L'apprentissage des auto-injections fut mené simultanément. Les sujets purent ensuite être inclus s'ils le souhaitaient dans une phase de traitement à domicile au cours de laquelle ils furent autorisés à pratiquer durant 6 semaines jusque 6 injections de la dose optimale préalablement déterminée de Moxisylyte ou d'AlprostadilAlfadex.

\section{Critères d'évaluation}

Le principal critère d'efficacité, au cours de la période au cabinet, fut l'obtention d'une rigidité pénienne axiale documentée par la capacité du pénis en érection à supporter une charge axiale de $1 \mathrm{~kg}$ sans que la hampe pénienne ploie («buckling test», fig. 1) [1, 10, 12].

$\mathrm{Au}$ cours de cette même période au cabinet, les critères d'efficacité secondaires furent l'évaluation du caractère adéquat de l'érection pour un rapport par l'investigateur et par le

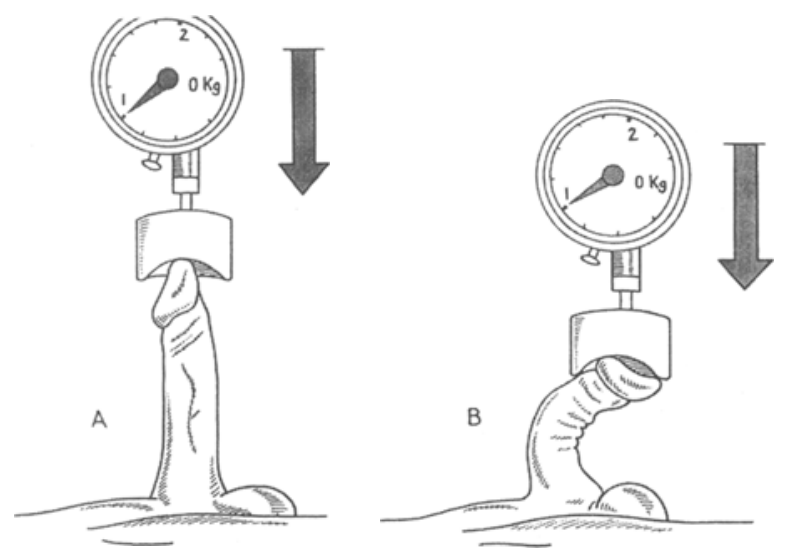

Figure 1 : Le buckling test : A : test positif : Penis avec rigidité axiale compatible avec un rapport sexuel ; $B$ : test négatif : Penis sans rigidité axiale suffisante pour un rapport. patient. Au cours de la phase de traitement à domicile, le principal paramètre d'efficacité fut la survenue d'érections rigides après autoinjection. Les critères secondaires furent le pourcentage de succès des auto-injections, et l'opinion du patient sur le traitement, ainsi que celle de sa partenaire. Les effets indésirables locaux furent répertoriés après chaque injection. Particulièrement, les sujets furent interrogés après chacune des érections soit seulement oralement (période au cabinet), soit à la fois oralement et par l'intermédiaire de leur journal d'auto-évaluation (période à domicile), à propos d'une éventuelle douleur pénienne, en faisant préciser si elle était survenue pendant l'injection, ou pendant l'érection, ou après elle.

\section{Méthodes}

Le buckling test fut pratiqué à l'aide d'un manomètre de pression étalonné en kgs, relié à une cape plastique rigide de $6 \mathrm{cms}$ de diamètre, concave sur sa face ventrale (fig. 1). En cas d'érection, l'investigateur appliqua la cape sur l'extrémité du pénis, le sujet étant en décubitus dorsal, en exerçant une pression vers le bas. Celle-ci fut lentement augmentée jusque atteindre la force de 1 kilogramme, en maintenant en permanence la pression vers le bas. Le test fut considéré positif si aucune déformation de la hampe pénienne n'apparaissait. Le buckling test fut répété à intervalles de $10 \mathrm{mn}$ jusque pendant 60 minutes, puis, si nécessaire, de nouveau à intervalles de $15 \mathrm{mn}$, en fonction de la durée de l'érection. L'évaluation clinique par le médecin du caractère adéquat de l'érection (compatible avec un rapport selon l'inspection et la palpation) se fit selon une échelle en 4 points dans laquelle le score 0 = pas de tumescence, 1 = tumescence partielle, 2 = tumescence complète avec rigidité partielle semblant compatible avec la pénétration, $3=$ rigidité complète. Les scores 2 et 3 furent considérés compatibles avec un rapport sexuel. Pendant la période à domicile, les sujets décrivirent les résultats de leurs injections dans des journaux d'évaluation. Il leur fut demandé de rapporter le nombre des injections ayant induit des érections adéquates pour un rapport sexuel. De plus, tant les patients que leur partenaire exprimèrent leur 
opinion du traitement au moyen d'une échelle analogique visuelle. Ils devaient tracer un trait coupant une ligne de $100 \mathrm{~mm}$ au niveau de laquelle 0 signifiait «le traitement ne me convient pas du tout» et 100 signifiait «le traitement me convient parfaitement». Leur score correspondit au nombre de $\mathrm{mm}$ séparant le trait qu'ils avaient tracé de l'extrémité 0 de cette ligne.

\section{Analyse statistique}

Elle utilisa le test exact de Fisher pour le buckling test, le test de Mann-Whitney pour l'évaluation de l'érection par l'investigateur au cours de la période au cabinet, le $\mathrm{Chi}^{2}$ pour les érections induites à domicile, le test $t$ de Student pour le pourcentage de succès des injections et les scores des échelles analogiques visuelles évaluant les opinions du patient et de sa partenaire sur le traitement, enfin le test exact de Fisher pour les effets indésirables.

\section{Médicaments étudiés}

Il s'est agi d'ampoules de $20 \mathrm{mg}$ de chlorhydrate de Moxisylyte lyophilisé (ICAVEX®) laboratoire LARGET $®)$ et d'ampoules de $20 \mu \mathrm{g}$ d'Alprostadil-Alfadex lyophilisé ((PGE1 $\alpha-c d$, CEDEX®, laboratoire Schwarz Pharma). Ces médicaments furent dissous dans un $\mathrm{ml}$ de sérum salé physiologique stérile. Pendant la phase en double insu au cabinet, les médications furent préparées par un pharmacien ou un médecin indépendant de l'étude. Les seringues de $2 \mathrm{ml}$ utilisées pour les 2 substances pendant les 2 périodes de l'étude furent prélevées dans les traitements d'ICAVEX, disponibles sur le marché.

\section{Population étudiée}

Cent quatre vingt six sujets avec dysfonction érectile datant d'au moins 6 mois ont été préinclus dans l'étude. Cent cinquante six remplissaient les critères d'inclusion et d'exclusion, et ont été répartis de façon randomisée entre Moxisylyte (81 cas) ou Alprostadil-Alfadex (75 cas). Le tableau 1 détaille les principales données démographiques. Les sujets avec dysfonction érectile d'origine primitivement hormonale, avec maladie de La Peyronie ou autre type de déformation du pénis, ainsi que ceux souffrant de maladies sévères, ont été exclus. L'âge moyen des patients était de 53,7 ans. L'origine de la dysfonction érectile était non-organique dans 73 cas (46\%), organique dans 41 cas ( $26 \%$ ) et mixte dans 43 cas $(28 \%)$. La durée moyenne de la dysfonction érectile était de 4,5 ans. Il n'a pas été trouvé de différence significative entre les groupes Moxisylyte et PGE1 $\alpha$-cd en ce qui concerne l'âge, l'origine de la dysfonction érectile, non plus que sa durée.

Tableau 1 : Caractéristiques démographies des populations étudiées

\begin{tabular}{lccc}
\hline & Moxisylyte & Alprostadil & Tous les sujets \\
\hline Nombre de sujets & 81 & 75 & 156 \\
\hline Age moyen (années) & 54,2 & 53,2 & 53,7 \\
$( \pm \mathrm{ET})$ & $(10,5)$ & $(11,1)$ & $(10,8)$ \\
\hline Cause de la dysfonction érectile & & & \\
Non-organique/psychogène & $35(43 \%)$ & $38(51 \%)$ & $73(46 \%)$ \\
Organique & $21(26 \%)$ & $20(27 \%)$ & $41(26 \%)$ \\
Mixte & $26(32 \%)$ & $17(23 \%)$ & $43(28 \%)$ \\
\hline $\begin{array}{l}\text { Durée de la dysfonction érectile } \\
\text { (années) : moyenne }( \pm \mathrm{ET})\end{array}$ & 4,3 & $\begin{array}{c}4,6 \\
(4,0)\end{array}$ & 4,5 \\
& & & $(5,2)$
\end{tabular}




\section{RESULTATS}

\section{Efficacité}

Cinquante six sujets du groupe AlprostadilAlfadex ( $75 \%$ ) et 32 du groupe chlorhydrate de Moxisylyte (40\%) eurent au moins un buckling test positif durant la période au cabinet (tableau 2 ). Lorsque les résultats furent répartis en fonction du type de dysfonction érectile, les proportions s'avérèrent, en ce qui concerne les patients principalement organiques, de 11/20 (55\%) après PGE1 $\alpha$-cd, et de 4/20 (20 \%) après Moxisylyte ; en ce qui concerne les sujets non organiques, ou psychogènes, de $32 / 38(84 \%)$ après PGE1 $\alpha$-cd, et 20/35 (57 \%) après Moxisylyte ; enfin, en ce qui concerne les patients mixtes, de 13/17 (76 \%) après PGE1 $\alpha$-cd, et de 6/20 (30 \%) après Moxisylyte. Quarante patients (53\%) eurent au moins 3 buckling tests positifs consécutifs, ce qui correspond à une érection totalement rigide d'au moins 20 minutes, après l'une au moins de leurs injections au cabinet, alors que la proportion ne fut que de $13(16 \%)$ après chlorhydrate de Moxisylyte. Les investigateurs jugèrent qu'au moins l'une des érections induites par l'Alprostadil-Alfadex était compatible avec un rapport sexuel chez 61 sujets (81 $\%$ ), contre 37 sujets (46\%) après chlorhydrate de Moxisylyte (sujets principalement organiques : PGE1 $\alpha$-cd 12/20 : $60 \%$, Moxisylyte 6/20 : $30 \%$; principalement non organiques : PGE1 $\alpha$-cd 34/38 : $89 \%$, Moxisylyte 19/35 : $54 \%$ ; mixtes : PGE1 $\alpha$-cd 15/17 : $88 \%$, Moxisylyte 12/26 : $46 \%$ ). Tous les critères d'efficacité évalués pendant la période au cabinet démontrèrent la supériorité de l'Alprostadil-Alfadex, avec un niveau de signification statistique $<0.001$. Huit sujets du groupe Moxisylyte (9\%) et un seul du groupe PGE1 $\alpha$-cd (1\%) abandonnèrent l'étude pendant la phase au cabinet pour raison d'inefficacité.

Soixante et un sujets du groupe Moxisylyte et 68 sujets du groupe Alprostadil souhaitèrent débuter la phase d'auto-injections à domicile. Cinquante huit sujets du groupe Alprostadil (85\%) rapportèrent avoir eu au moins une érection rigide, contre $37 \mathrm{du}$ groupe Moxisylyte $(61 \%)$ (tableau 3$)$. Le pourcentage de succès moyen des auto-injections fut de $61 \%$ dans le groupe PGE1a-cd, contre $44 \%$ dans le groupe chlorhydrate de Moxisylyte $(\mathrm{p}<0.05)$. L'opinion du sujet sur son traitement ainsi que celle de sa partenaire atteignirent des scores significativement supérieurs dans le groupe Alprostadil-Alfadex par rapport au groupe Moxisylyte (tableau 3).

\section{Tolérance}

Les données apparaissent dans les tableaux 4 et 5. Pendant la période au cabinet, la douleur du pénis fut l'effet indésirable local le plus fréquent. L'incidence de la douleur pendant l'injection fut la même dans les 2 groupes. La fréquence des douleurs accompagnant l'érection fut significativement plus importante dans le groupe Alprostadil. Il ne survint d'érection prolongée, définie par une durée supérieure à 2 heures, que dans le groupe Alprostadil. Une seule dépassa 3 heures ( $3 \mathrm{~h} 15$ ).

Pendant la période d'auto-injections à domicile, la douleur fut également l'effet indésirable le plus souvent rapporté. De nouveau les phénomènes douloureux survenant pendant ou après l'érection furent significativement plus fréquents dans le groupe PGE1 $\alpha$-cd, tandis que les incidences des douleurs pendant l'injection, des saignements locaux, et des érections prolongées, ne montrèrent pas de différence statistiquement significative. Les sujets du groupe Moxisylyte rapportèrent plus souvent des sensations ébrieuses ou à type d'hypotension.

\section{DISCUSSION}

Plusieurs études ouvertes ont montré que le chlorhydrate de Moxisylyte, qui est un bloqueur des récepteurs alpha-1 adrénergiques, entraînait moins d'érections complètes que la papavérine ou la PGE1 lorsqu'on l'injectait dans les corps caverneux [3, 4, 5]. Le Moxisylyte s'est cependant montré supérieur au placebo dans des études en double insu, et c'est dans les dysfonctions érectiles à prédominance neurogène et psychogène qu'il donne ses meilleurs résultats $[4,5,7,14]$. Ce sont les doses de 10 à $20 \mathrm{mg}$ qui sont les plus efficaces, sans bénéfice supplémentaire de la dose de 30 mg [14]. Le Moxisylyte a été présenté comme une substance facilitant l'érection, dont l'effet est meilleur à domicile, lorsque l'injection peut 


\begin{tabular}{lcccc}
\hline & Moxisylyte & Alprostadil & Test & Valeur duP \\
\hline Nombre de sujets & 81 & 75 & & \\
\hline $\begin{array}{l}\text { Sujets avec buckling test } \\
\text { Positif 1 }\end{array}$ & 32 & 56 & Fisher & $<0.001$ \\
\hline $\begin{array}{l}\text { Sujets avec 3 buckling test } \\
\text { consécutifs positifs 2 }\end{array}$ & 13 & $(75 \%)$ & & \\
\hline $\begin{array}{l}\text { Sujets avec érection adéquate } \\
\text { pour un rapport (évaluation par }\end{array}$ & $(16 \%)$ & 40 & Fisher & $<0.001$ \\
l'investigateur) 3 & $(46 \%)$ & 61 & & \\
& & $(81 \%)$ & Mann-Whitney & $<0.001$ \\
\hline
\end{tabular}

1. Au moins un buckling test positif à l'unes des sessions au cabinet (période 1)

2. Trois buckling tests positifs consécutifs repésentant un érection rigide de $20 \mathrm{mn}$ à au moins un des sessions au cabinet (période 1)

3. Meilleure réponse obtenue pendant les 3 sessions au cabinet (période 1)

\section{Tableau 3 : Réponse érectile au cours du traitement par auto-injections à domicile}

\begin{tabular}{|c|c|c|c|c|}
\hline & Moxisylyte & Alprostadil & Test & Valeur duP \\
\hline Nombre de sujets & 61 & 68 & & \\
\hline $\begin{array}{l}\text { Sujets avec au moins une érection } \\
\text { rigide après une auto-IC }\end{array}$ & $\begin{array}{c}37 \\
(61 \%)\end{array}$ & $\begin{array}{c}58 \\
(85 \%)\end{array}$ & Chi 2 & $<0.001$ \\
\hline $\begin{array}{l}\text { Pourcentage moyen des succès } \\
\text { après auto-IC }( \pm \mathrm{ET})\end{array}$ & $\begin{array}{c}43,7 \% \\
( \pm 41,1 \%)\end{array}$ & $\begin{array}{l}61,2 \% \\
( \pm 38,5)\end{array}$ & t-test & $<0.05$ \\
\hline $\begin{array}{l}\text { Opinion du sujet à propos de son } \\
\text { traitement } 1 \text { (score moyen en mn, } \\
\pm \text { ET) }\end{array}$ & $\begin{array}{c}36,1 \\
( \pm 25,8)\end{array}$ & $\begin{array}{c}52,8 \\
( \pm 27,6)\end{array}$ & t-test & $<0.001$ \\
\hline $\begin{array}{l}\text { Opinion de la partenaire à propos } \\
\text { du traitement } 1 \text { (score moyen en } \\
\text { mn, } \pm \text { ET) }\end{array}$ & $\begin{array}{c}36,7 \\
( \pm 26,6)\end{array}$ & $\begin{array}{c}49,9 \\
( \pm 28,5)\end{array}$ & t-test & $<0.01$ \\
\hline
\end{tabular}

1. Score analogique visuel de 0 à $100 \mathrm{mn}$, où 0 équivaut à «ce traitement ne me convient pas du tout» et 100 équivaut à «ce traitement me convient parfaitement».

Tableau 4 : Nombre de sujets avec effet indésirable local pendant la période au cabinet

\begin{tabular}{lccc}
\hline Effets indésirables & Moxisylyte & Alprostadil & Valeur du P (Fischer) \\
\hline Douleur pendant l'injection & $12(15 \%)$ & $10(13 \%)$ & $\mathrm{NS}$ \\
Douleur pendant l'érection & $2(3 \%)$ & $13(\mathbf{1 7 \% )}$ & $<0.001$ \\
Douleur après l'érection & $0(0 \%)$ & $5(7 \%)$ & $<0.05$ \\
\hline Saignement & $2(3 \%)$ & $2(3 \%)$ & $\mathrm{NS}$ \\
\hline Erection $>2$ heures & $0(0)$ & $4(5 \%)$ & $\mathrm{NS}$
\end{tabular}


Tableau 5 : Nombre de sujets avec effet indésirable local au cours de la période d'auto-injections à domicile

\begin{tabular}{lccc}
\hline Effets indésirables & Moxisylyte & Alprostadil & Valeur du P (Fischer) \\
\hline Douleur pendant l'injection & $9(15 \%)$ & $17(25 \%)$ & $\mathrm{NS}$ \\
Douleur pendant l'érection & $3(5 \%)$ & $16(24 \%)$ & $<0.001$ \\
Douleur après l'érection & $3(5 \%)$ & $13(19 \%)$ & $<0.05$ \\
\hline Saignement & $3(5 \%)$ & $10(15 \%)$ & $\mathrm{NS}$ \\
\hline Erection > 2 heures & $1(2)$ & $3(4 \%)$ & $\mathrm{NS}$ \\
\hline Sensation ébrieuse/hypotension & $5(8 \%)$ & $\mathbf{1 ( 1 \% )}$ & $\mathrm{NS}$
\end{tabular}

être associée à une stimulation sexuelle, dans l'environnement habituel du patient [5].

Il a été rapporté que l'Alprostadil induit une rigidité complète chez environ $70 \%$ des sujets avec dysfonction érectile de toutes origines lorsqu'on l'injecte au cabinet dans les corps caverneux [11, 13, 15]. Comme pour le Moxisylyte, la stimulation sexuelle à domicile augmente le taux des réponses positives. L'Alprostadil a été utilisé à des doses de 1 à $80 \mu \mathrm{g}$. La majorité des patients répond cependant à des doses inférieures à $40 \mu \mathrm{g}[11,15]$.

Notre étude est la première à avoir comparé de façon randomisée 2 substances ayant obtenu l'autorisation de mise sur le marché dans l'indication de la dysfonction érectile. Du fait de la différence d'aspect des emballages des médicaments étudiés, seule la période au cabinet peut être considérée totalement aveugle. Il a fallu recourir à une tierce personne indépendante pour pouvoir cacher au sujet et au médecin le type de substance injectée. Pendant cette période au cabinet, l'efficacité de l'Alprostadil-Alfadex s'avéra significativement supérieure à celle du chlorhydrate de Moxisylyte dans chaque catégorie étiologique. La proportion des sujets principalement organiques ayant obtenu une érection rigide au cabinet, selon le résultat du buckling test, fut particulièrement faible après Moxisylyte $(20 \%$ contre $55 \%$ après $\mathrm{PGE} 1 \alpha-\mathrm{cd})$. Huit sujets du groupe Moxisylyte interrompirent l'étude à ce stade pour manque d'efficacité, contre un seul $d u$ groupe PGE1 $\alpha-c d$. Pendant la phase des autoinjections, le taux de succès du Moxisylyte augmenta. Cependant, l'Alprostadil-Alfadex resta significativement plus efficace. Six nouveaux sujets interrompirent l'étude pour inefficacité dans le groupe Moxisylyte (10\%) contre $3(4 \%)$ dans le groupe Alprostadil-Alfadex.

L'incidence des effets indésirables liés à l'injection elle-même (douleur pendant l'injection, saignement) fut identique dans les 2 groupes. Le Moxisylyte induisit un peu plus d'effets indésirables de type cardio-vasculaire comme hypotension ou les sensations ébrieuses. Par contre, les phénomènes douloureux pendant et après l'érection, et les érections prolongées $(>2 \mathrm{~h}$ ), furent plus fréquents dans le groupe Alprostadil-Alfadex. Ces incidences d'effets indésirables sont en bon accord avec ce qui a déjà été rapporté pour les 2 substances [3, $4,5,7,13,15]$. Par contre, cette étude ne peut fournir d'information sur le risque de fibrose des corps caverneux du fait du faible nombre d'injections et de la durée brève de la phase à domicile. Si l'efficacité de l'Alprostadil-Alfadex est manifestement supérieure à celle du Moxisylyte, sa tolérance est donc légèrement moins bonne du fait d'une fréquence plus élevée d'érections douloureuses et prolongées. Cette différence semble cependant peu conséquente, puisque seulement 4 sujets du groupe Alprostadil (5.3\%) interrompirent le traitement pour effet indésirable lié à celui-ci, incluant 2 pour érection prolongée, et 2 pour douleur, 2 l'ayant interrompu pour la même raison dans le groupe Moxisylyte $(2.5 \%)$, l'un pour douleur et l'autre pour sensations ébrieuses. 
Tant la douleur que les érections prolongées sont généralement dose-dépendantes, et disparaissent en diminuant la dose $[4,13,15]$. La douleur disparaît même souvent en répétant les injections à la même dose, une fois surmontée l'anxiété suscitée chez les patients par cette sensation inhabituelle. Beaucoup d'entre eux préfèrent d'ailleurs ressentir quelques sensations un peu désagréables et avoir des érections fortes, plutôt que d'avoir des érections indolores mais seulement partielles. Le degré de satisfaction du traitement, qui reflète à la fois son efficacité et sa tolérance, fut d'ailleurs significativement plus élevé avec l'Alprostadil-Alfadex, aussi bien chez les sujets que chez leur partenaire.

\section{CONCLUSIONS}

L'Alprostadil-Alfadex est significativement plus efficace que le chlorhydrate de Moxisylyte en ce qui concerne l'induction d'érections rigides, aussi bien au cabinet qu'à domicile. Les effets indésirables liés à l'injection surviennent avec la même fréquence avec les 2 substances, tandis que le Moxisylyte induit plus d'effets indésirables systémiques et la Prostaglandine plus d'érections douloureuses et prolongées. Tant les sujets que leur partenaire sont significativement plus satisfaits de l'Alprostadil-Alfadex.

\section{Remerciements :}

\section{A Mlle Nathalie BARLET pour la préparation du manuscrit.}

\section{REFERENCES}

1. ALLEN R.P., SMOLEV J.K., ENGEL R.M., BRENDLER C.B. : Comparison of rigiscan and formal nocturnal penile tumescence testing in the evaluation of erectile rigidity. J. Urol., 1993, 149 : 1265

2. BRINDLEY G.S. : Cavernosal Alpha-Blockade : A new technique for investigating and treating erectile impotence. Brit. J. Psychiat. , 1983, 143 : 332

3. BUVAT J., BUVAT-HERBAUT M., LEMAIRE A. AND MARCOLIN G. : Reduced rate of fibrotic nodules in the cavernous bodies following auto-intracavernous injection of Moxisylyte compared to papaverine. Int. J. Impotence Res., 1991, 3 : 123

4. BUVAT J., LEMAIRE A. AND BUVAT-HERBAUT M. : Intracavernous pharmacotherapy : comparison of
Moxisylyte and Prostaglandin E1. Int. J. Impotence Res., 1996, $8: 41$

5. BUVAT J., LEMAIRE A., BUVAT-HERBAUT M. AND MARCOLIN G. : Safety of intracavernous injections using an alpha-blocking agent. J. Urol. , 1989, 141 : 1364

6. COSTA P., SARRAZIN B., BRESSOLLE F., COLSON M.H., BONDIL P. AND SAUDUBRAY F. : Efficiency and side effects of intracavernous injections of Moxisylyte in impotent patients : a dose-finding study versus placebo. J. Urol. , 1993, $149: 301$

7. COSTA P., SARRAZIN B., BRESSOLLE F., MOTTET N., LOUIS J.F., SAUDUBRAY F. AND NAVRATIL $H$.: Is the volume injected a parameter likely to influence the erectile response observed after intracavernous administration of an alpha-blocking agent ? Eur. Urol., 1993, $24: 43$

8. ISHII N., WATANABE H., IRISAWA C; KIKUCHI Y., KAWAMURA S.AND SUZUKI K. : Studies on male sexual impotence, Report 18. Therapeutic trial with Prostaglandin E1 for organic impotence. Nippon Hinyokika Gakkai Zasshi, 1986, 77 : 954

9. A JUENEMAN K.P AND ALKEN P. : Pharmacotherapy of erectile dysfunction : A review. Int. J. of Impotence Res. , 1989, $1: 71$

10. KARACAN I., MOORE C. AND SAHMAY S. : Measurement of pressure necessary for vaginal penetration. Sleep Res., 1985, 14 : 269

11. LEA A.P., BRYSON H.M. AND BALFOUR J.A. : Intracavernous alprostadil : A review of its pharmacodynamic and pharmacokinetic properties and therapeutic potential in erectile dysfunction. Drug \& Aging, 1996, $8: 56$

12. LICHT M.R., LEWIS R.W., WOLLAN P.C. AND HARRIS C.D. : Comparison of rigiscan and sleep laboratory nocturnal penile tumescence in the diagnosis of organic impotence. J. Urol., 1995, 154 : 1740

13. LINET O.I. AND OGRINC F.G. : Efficacy and safety of intracavernosal alprostadil in men with erectile dysfunction. New Engl. J. Med., 1996, 334 ; No. 14 : 873

14. NAVRATIL H., COSTA P., LOUIS J.F., ANDRO M.C. AND SAUR P. : Efficacité et tolérance du Moxisylyte en injection intracaverneuse chez le patient présentant un dysfonctionnement érectile : relation effet/dose versus placebo. Progrès en Urologie, 1995, 5: 690

15. PORST H. : The rationale for Prostaglandin E1 in erectile failure : A survey of worldwide experience. J. Urol., 1996, 155 : 802

16. PORST H., VAN AHLEN H., BLOCK T., HALBIG W., HAUTMANN R., L^CHNER-ERNST D., RUDNICK J., STAEHLER G., WEBER W.M., WEIDNER W., WEISKE W.H. : Intracavernous self-injection of Prostaglandin E1 in the therapy of erectile dysfunction. VASA, 1989, Suppl. $28: 50$

17. VIRAG R. : Intracavernous injection of papaverine 
Conclusions : Alprostadil Alfadex is significantly more effective than Moxisylyte chlorhydrate in producing full penile rigidity in office and at home. Injection related side

\section{ABSTRACT}

Double-blind multicenter study comparing Alprostadil Alfadex with moxisylyte chlorhydrate in patients with chronic erectile dysfunction

\section{J. Buvat, E. Amar, O.Cambau , P.Desvaux, A. Gout, A. LeRICHE}

Purpose : To compare the efficacy and safety of Alprostadil Alfadex (PGE1a-cd) and Moxisylyte - chlorhydrate to induce reactions adequate for sexual intercouse in a prospective, randomized, parallel double blind study in patients with erectile dysfunction.

Methods : 156 patients with erectile dysfunction due to organic, non-organic and mixed origin were randomized into two paralle treatment groups receiving titrations of their individual optimum dose of either Alprostadil Alfadex or Moxisylyte chlorhydrate. Erectile response was measured by the buckling test. A positive test was associated with an axial erection rigidity that did not buckle/deform to $1.0 \mathrm{~kg}$ load. The buckling test was repeated every 10 minutes for up to 60 minutes.

Results : 56 patients (75\%) in the Alprostadil Alfadex group and 32 patients $(40 \%)$ in the Moxisylyte chlorhydrate group responded with at least one positive buckling test during the office period. The investigators assessed erections following. Alprostadil Alfadex to be adequate for sexual intercourse in 61 patients $(81 \%)$ compared to 37 patients (46\%) following Moxisylyte chlorhydrate. All efficacy parameters in offeice reached statistical significance of $p<0.001$ in favour of Alprostadil Alfadex. During self-injection therapy at home 58 patients $(85 \%)$ reported at least one rigid erection following Alprostadil Alfadex compared to 37 patients $(61 \%)$ after Moxisylyte chlorhydrate. Patient's and partner's opinion of the treatment achieved statistically significant better scores in the Alprostadil Alfadex group compared to the Moxisylyte chlorhydrate group. effects occur with the same frequency while Moxisylyte results in more systemic side effects and PGE1 is related with more painful and prolonged erections. Both the patients and their partners significantly more satisfied with the use of Alprostadil Alfadex.

Key words : Impotence, erectile dysfunction, buckling test, auto-injections, Alprostadil Alfadex, Moxisylyte - chlorhydrate, Prostaglandine E1. 